INPLASY

PROTOCOL

To cite: Bai et al. Benefits and risks of rapid initiation of antiretroviral therapy: A systematic review and metaanalysis. Inplasy protocol 202210004. doi:

10.37766/inplasy2022.1.0004

Received: 01 January 2022

Published: 01 January 2022

Corresponding author:

Lili Dai

lilydaier@ccmu.edu.cn

Author Affiliation:

Center for Infectious Diseases, Beijing Youan Hospital, Capital

Medical University.

Support: National 13th FiveYear Grand.

Review Stage at time of this submission: Data extraction.

\section{Benefits and risks of rapid initiation of antiretroviral therapy: A systematic review and meta-analysis}

Bai, RJ1; Lu, SY2; Dai, L³.

Review question / Objective: This systematic review and meta-analysis aimed to evaluate the benefits and risks of Rapid ART compared to standard/delayed treatment, and to provide a reliable reference for the selection of active treatment.

Condition being studied: According to the World Health Organization (WHO), an estimated 37.7 million people worldwide were living with HIV in 2020. The use of antiretroviral therapy (ART) can effectively reduce HIV mortality and transmission. These findings support WHO recommendations for accelerated initiation of ART compared to standard or delayed treatment, with rapid ART when medical conditions and the patient's physical condition permit.

INPLASY registration number: This protocol was registered with the International Platform of Registered Systematic Review and Meta-Analysis Protocols (INPLASY) on 01 January 2022 and was last updated on 01 January 2022 (registration number INPLASY202210004).

Conflicts of interest:

None declared.

\section{INTRODUCTION}

Review question / Objective: This systematic review and meta-analysis aimed to evaluate the benefits and risks of Rapid ART compared to standard/delayed treatment, and to provide a reliable reference for the selection of active treatment.

Condition being studied: According to the World Health Organization (WHO), an estimated 37.7 million people worldwide were living with HIV in 2020. The use of antiretroviral therapy (ART) can effectively 
reduce HIV mortality and transmission. These findings support WHO recommendations for accelerated initiation of ART compared to standard or delayed treatment, with rapid ART when medical conditions and the patient's physical condition permit.

\section{METHODS}

Search strategy: We will search the EMBASE, The Cochrane Library, PubMed, Web of science from the date of inception for randomized controlled trials without language restriction.

\section{Participant or population: Patient with HIV.}

Intervention: The intervention group was treated with Rapid ART (immediate/sameday diagnosis and treatment).

Comparator: The control group was treated with standard/delayed treatment.

Study designs to be included: Cohort study; RCT.

Eligibility criteria: Literature inclusion criteria: (1) The intervention group was treated with Rapid ART (immediate/sameday diagnosis and treatment), while the control group was treated with standard/ delayed treatment; (2) Articles in English only; (3) No restrictions on gender, age and region.

Information sources: The literature was retrieved from English databases of PubMed, Cochrane Library, Embase and Web of Science.

Main outcome(s): The outcome measures of this meta-analysis were viral load $<100$ copies $/ \mathrm{mL}$ at 12 months after treatment, continuing care, mortality.

Additional outcome(s): Adverse events, infected tuberculosis, and severe bacterial infections.

Quality assessment / Risk of bias analysis: Cochrane Collaboration Risk of Bias Tool.
Strategy of data synthesis: Meta-analysis was performed on the data with Stata15.0 software. In this Meta-analysis, the combined effect sizes of viral load $<100$ copies/mL, continuing care, mortality, adverse events, infected tuberculosis, severe bacterial infections were expressed by the relative risk rate (RR) and its $95 \%$ confidence interval, namely RR $(95 \% \mathrm{Cl})$. Q test and 12 were used to quantify the heterogeneity among different studies. When I20.05, a fixed-effect model was used to combine the outcome measures; and when $12 \geq 50 \%$ or $\mathrm{P}<0.05$, a random-effect model was adopted to combine the outcome measures.

Subgroup analysis: State any planned investigation of 'subgroups'. Be clear and specific about which type of study or participant will be included in each group or covariate investigated. State the planned analytic approach. To be determined based on the literature retrieved.

Sensitivity analysis: Publication bias will be assessed by a funnel plot for meta-analysis and quantified by the Egger method.

Country(ies) involved: China.

Keywords: HIV; Antiretroviral; Rapid; Metaanalysis.

Contributions of each author:

Author 1 - RJ Bai.

Email: bairuojing163@163.com

Author 2 - SY Lu.

Email: a2068154965@163.com

Author 3 - Lili Dai.

Email: lilydaier@ccmu.edu.cn 